\title{
Dialogue sur la poésie de Louis Le Caron: Commentaire*
}

\author{
SHARON A. ADAMS et CHARLES JOSE
}

Publié en 1556, le dialogue de Le Caron, Ronsard. ou, de la Poësie, offre à la fois un exemple-clef d'un genre qui attire de plus en plus l'attention des seiziémistes et une mise au point précise des rapports entre la philosophie néo-platonicienne et les revendications de la poésie. Ceci se passe à une époque où les jeunes poètes de la Pléiade se trouvaient appuyés dans leur ambition avouée de rivaliser avec les grands poètes de l'antiquité, sinon de les surpasser. Louis Le Caron voulait, avec ce dialogue, se placer parmi leurs admirateurs et approuver leurs aspirations patriotiques, tout en exprimant des réserves d'ordre philosophique.

En 1556 Le Caron, connu aussi sous le nom de Charondas, n'avait que vingt-deux ans. ${ }^{1}$ Mais il était avocat au Parlement de Paris depuis 1552. Il était déjà l'auteur d'un certain nombre de livres - notamment un volume d'annotations faites à une oeuvre juridique en latin $;^{2}$ un recueil de sonnets et d'odes en $1554 ; ;^{3}$ un dialogue sur le droit, paru la même année; ${ }^{4}$ et un petit exposé sur la philosophie platonicienne, suivi d'un dialogue où il démontre la nécessité de fonder tout bon gouvernement sur des principes proprement philosophiques. ${ }^{5}$ Avec les Dialogues de 1556 où figure le Ronsard, Le Caron a voulu continuer à se bâtir une renommée dans les cercles philosophiques et littéraires. La table des matières annonce trois livres de dialogues dont les titres portent tous sur des questions relatives à la philosophie. Cependant, le projet du jeune parlementaire est resté inachevé, malgré la promesse contenue dans la liste de dialogues et renouvelée à la dernière page. ${ }^{6}$ Le Caron a préféré poursuivre sa carrière de jurisconsulte. Avant de mourir en 1613 à Clermont en Beavoisis, où il avait rempli les fonctions de lieutenant du bailliage entre 1567 et 1593 , il avait publié en latin et en français de nombreuses oeuvres juridiques (surtout sur le droit romain et le droit coutumier parisien), qui lui ont valu la célébrité qu'il n'avait pas connue de son vivant en tant que poète et philosophe.

* Notre transcription du texte de ce dialogue a paru dans Renaissance and Reformation/Renaissance et Réforme, nouvelle série 3 (1979), 132-158. 
On ne sait pas pourquoi Le Caron n'a pas donné suite au projet ambitieux annoncé dans les Dialogues. Cependant, une lecture de la table des matières nous permet de spéculer sur les intentions encyclopédiques de l'auteur. Brian Barron a essayé d'élucider le sens du dialogue sur la poésie en examinant ses rapports avec le projet envisagé et les dialogues qui l'entourent. ${ }^{7}$

La table des matières se présente ainsi:

\section{Dialogues du premier livre}

Le Courtisan premier, Ou, que le Prince doit philosopher.

Le Courtisan second, Ou de la vraie sagesse, et des louanges

de la philosophie.

Valton, de la tranquilité d'Esprit, Ou du souverain bien.

Ronsard, ou de la poësie.

Claire, ou de la beauté iiii dialo. desquelz le premier est

comme l'argument, ou epitome des autres.

\section{Du second livre.}

Le Chaldéan, Ou des divinations.

Pasquier, ou l'Orateur.

Le Solitaire, ou de la description du monde.

Le Sophiste, ou de la science.

Faulchet, ou de l'utilité, qu'apporte la congnoissance des choses naturelles

ii. dia.

ii. dia.

iiii. dia.

ii. dia.

Du tiers livre.

Le nouveau Narcisse, Ou de la nature de l'homme.

ii. dia.

Le nouveau Heraclite, ou des secrets de la philosophie non encores congneus ne revelez.

Le nouveau Parmenide, ou de l'Estant, et des Idées.

Le nouveau Pytagore, ou des nombres et de l'harmonie.

Le Senateur, ou de la Chosepublique

iii. dia.

vi. dia.

x. dia.

Le premier livre, et le seul qui ait été publié, traite surtout de l'utilité de la philosophie dans les domaines public et privé. Dans le deuxième livre il aurait été question plutôt de philosophie naturelle; dans le troisième, de métaphysique platonicienne en rapport avec l'hermétisme pythagoricien. Et le tout aurait précédé une dernière série de dialogues (dix, comme chez Platon) sur la Chose publique. Le dialogue sur la poésie aurait donc figuré vers le début de ce qui porte toutes les marques d'une apologie de la philosophie, apologie où l'auteur aurait tenté de traiter, dans une perspective humaniste, les questions essentielles que cette science se pose. Lorsqu'on considère de plus près le premier livre, on perçoit qu'à la fin du troisième dialogue, Valton, de la tranquilité d'Esprit, Ou du souverain bien, qui précède celui sur la poésie, la vraie tranquillité serait purement intellectuelle. Valton, à qui l'auteur accorde le dernier argument et qui porte le nom de famille de la mère de Le Caron, conclut: 
L'aise donc de l'esprit non pertroublé surpasse toutes les voluptez... Quelle plus grande joie du repos et contentement peut avoir l'homme, que de jouir de soi-mesme, c'est à dire du repos et contentement lequel il se donne par les pensées, conceptions et discours, qu'il fait en lui mesme des choses excellentes et louables. (126)

Il semble en effet régner dans tout ce premier livre des Dialogues un désir de centrer l'attention des interlocuteurs et du lecteur sur les implications morales des problèmes philosophiques abordés. On reviendra à ces observations contextuelles après avoir considéré la forme du dialogue adoptée par Le Caron dans son Ronsard et les arguments de chaque interlocuteur.

De récentes études ont essayé de commenter l'importance de la forme dialoguée pendant la Renaissance. ${ }^{8}$ En décrivant les dialogues de Le Caron, M. Bénouis constate qu'en général ce ne sont pas de vrais procédés dialectiques qui gouvernent leur déroulement interne, mais plutôt celui de la question-réponse et celui du pro et contra. ${ }^{9}$ Eva Kushner élargit encore le problème du choix de cette forme par les vulgarisateurs de la philosophie au XVIe siècle, et son article nous permet d'apporter des nuances au jugement de Bénouis. Pour elle "le dialogue constitue à la Renaissance une forme privilégiée d'échange intellectuel, particulièrement accordée aux besoins épistémologiques de l'époque ..., car il implante la possibilité d'une présence de l'Autre dans le discours." ${ }^{10} \mathrm{La}$ forme même du dialogue peut refléter, chez ceux qui ont pratiqué ce genre, un désir de chercher de nouvelles synthèses intellectuelles à un moment historique (du moins avant la Réforme et la Contre-Réforme) favorable à la réalité des opinions de l'Autre. Avec son analyse structuraliste de plusieurs dialogues, Eva Kushner propose un critère génologique moyennant lequel on pourrait juger du degré de "dialogicité" d'un texte disposé en dialogue. On pourrait dire qu'un tel texte assume sa "dialogicité" dans la mesure où "l'autre intervient d'une manière effective", plutôt que d'être le porte-parole des objections que le scripteur sait prévoir et réfuter, ou de jouer tout simplement le rôle d'un "résonnateur" à celui qui semble détenir la vérité. ${ }^{11}$ Il s'agirait donc de déterminer l'effet de chaque interlocuteur sur le message qu'on peut déduire du texte entier. Vu dans cette perspective, le Ronsard de Le Caron a mieux assumé sa "dialogicité" si on le compare au premier dialogue, le Courtisan. Car dans celui-ci Le Caron parle en son propre nom, et Philarète, le seul autre interlocuteur (d'ailleurs fictif), joue plutôt le rôle de disciple; tandis que dans le Ronsard Le Caron répartit des opinions opposées sur la nature et la fonction de la poésie parmi quatre interlocuteurs, sans tirer de conclusions bien nettes. ${ }^{12}$ La présence de l'Autre dans ce dialogue entraîne la possibilité d'un véritable 
échange d'idées, et d'une dialectique à la recherche d'une vérité plus complexe, même si cette possibilité reste plutôt virtuelle.

Le choix de la forme dialoguée et des noms des interlocuteurs n'a rien d'arbitraire. Au début du dialogue Le Caron signale au lecteur qu'il veut "expliquer" ses idées sur la nature de la poésie "en manière de familier devis". A l'exemple des anciens il veut "embellir et decorer de toutes ses bienseances" son dialogue, en faisant discourir sur la dignité de la poésie "les deux, qui sont au jourd'hui à bon droit reputez les premiers poëtes de nostre tems, Ronsard et Jodelle, avec deux orateurs Pasquier et Fauchet, lesquelz l'excellence de leur esprit, pour la bonne esperance d'eux m'a fait tousjours aimer" (128). Il cherche donc comme les poètes de la Pléiade à imiter les anciens et à faire oeuvre d'art à son tour en invoquant le critère interne de la bienséance. En même temps il souligne le caractère fictif du dialogue et essaie de parer à toute accusation de déformation de pensée: "je croi, que se resouvenant de la coustume des dialogues [aucun des interlocuteurs] ne trouvera estrange, que j'aie emprunté son nom et sa personne" (128v). Le Caron semble vouloir que l'on juge son dialogue selon ses propres mérites artistiques.

En 1556, au moment de la publication des Dialogues, Ronsard était connu comme le premier poète lyrique de France, auteur des Odes (1550, 1552), des Amours (1552) et de la Continuation des Amours (1555), et des Hymnes (1555). Avec la représentation de sa Cléopâtre captive au Collège de Boncourt en 1552, Jodelle commençait à jouir d'une réputation de grand poète tragique. Estienne Pasquier (1529-1615) et Claude Fauchet (1530-1602), futurs historiens des lettres françaises, étaient déjà admis au barreau de Paris, et appartenaient comme Le Caron au monde des parlementaires. Comme lui aussi ils tenaient à faire figure dans les cercles humanistes et littéraires. ${ }^{13}$ Pasquier avait publié en 1554 son Monophile, dialogue dans lequel trois jeunes gens et une dame, aux noms fictifs, discutent des problèmes de l'amour. Les rapports de Pasquier avec Ronsard sont documentés dans une lettre que ce premier lui adresse en 1555 , où il se montre content que son nom paraisse dans l'oeuvre du poète. Tout en louant les écrits de Ronsard, Pasquier n'hésite pas à ajouter des réserves:

... pour vous dire en amy, je trouve tres beaux [vos escrits] lorsqu'avez seulement voulu contenter vostre esprit: mais quand par une servitude à demy courtisane estes sorty de vous mesmes pour estudier au contentement tantost des grands tantost de la populace, je ne les trouve de tel alloy. ... .je souhaiterois que ne fissiez si bon marché de vostre plume à hault-louër quelques-uns que nous savons notoirement n'en estre dignes. ${ }^{14}$

Dans ses Recherches de la France Pasquier consacre quelques lignes à Le Caron dont le nom paraît dans une liste de poètes célèbres. ${ }^{15}$ Cependant, ni Ronsard ni Jodelle ne semblent avoir fait à Le Caron l'honneur 
d'immortaliser son nom dans leurs poèmes. Toujours est-il que Le Caron, à la suite de Platon, a choisi des noms de contemporains dont deux étaient de ses amis. Et, selon le critère d'Eva Kushner, ce souci est "directement lié, par son intentionalité du moins, à la quête dialogique de la vérité, autrui étant représenté dans le texte par un personnage (ou plusieurs) dont l'altérité est garantie par l'historicité ou par la vraisemblance." ${ }^{16}$ En même temps, en les désignant comme poètes et orateurs, Le Caron installe dans le dialogue une dichotomie potentielle entre les buts de la poésie et ceux de la rhétorique, bien que ces deux domaines soient souvent étroitement liés comme arts de communication dans les écrits théoriques de l'époque, y compris la Deffence de Du Bellay. ${ }^{17}$

Quoique la description du cadre soit réduite à quelques phrases, le choix du Palais Royal de Paris, où "les compagnies des hommes doctes ...devisent quelquefois de choses graves et serieuses" (128v), fournit au dialogue un cadre célèbre et approprié. Le ton de "familier devis" s'établit au début avec une évocation du "bruit de tant de voix tumultuantes", bruit qui va faire l'objet des commentaires des interlocuteurs. ${ }^{18}$ Ronsard contraste son effet sur l'oreille avec celui des "amoureuses douceurs" de la poésie. Pasquier rétorque que, pour ceux qui fréquentent le Palais Royal, "l'accoustumance conjointe avec un gracieux espoir du gain" ont transformé le bruit du palais de justice en "plaisir" (128v). Exemple de la sophistique de l'orateur? Ou de l'esprit accommodant de Pasquier qui va jouer plutôt le rôle de conciliateur dans le dialogue? Un échange d'éloges courtois entre les deux poètes termine cette petite mise en scène.

Le caractère proprement philosophique et élitiste de la poésie est signalé dès la première phrase du dialogue. Le Caron identifie la poésie comme une "science" qui a servi à d'autres nations de "commencement" pour parvenir "à la gloire de la souveraine congnoissance de toutes les choses lesquelles l'homme bien né doit embrasser" (127-127v). Citant Platon à son appui, il précise que "de sa nature la poësie est pleine d'enigmes, et non vulgaire à châcun". Cet hermétisme est voulu par les anciens, qui "rendoient [la poësie] admirable par une grave obscurité de sentences" (127v). Comme Michel Foucault l'a si bien montré, toute écriture, philosophique ou poétique, jusqu'à la fin du XVIe siècle repose sur le concept de la ressemblance, et porte les marques de la conscience d'une vérité ultime mais cachée. ${ }^{19} \mathrm{Ce}$ serait donc la fonction de l'écrivain de capter et de reproduire sous forme de langue les analogies qui sont la condition a priori de l'existence de l'homme et des choses dans une seule et souveraine nature. $\mathrm{Au}$ milieu du siècle les humanistes qui cherchaient à expliciter cette façon de concevoir l'homme et le monde avaient à leur disposition tout un corpus de textes qui inclut les dialogues de Platon, le commentaire de Marsile Ficin sur ces dialogues, les écrits qui constituent la théologie ancienne ou la prisca theologia, la patrologie 
grecque et tous les ouvrages des apologistes d'Alexandrie qui proposaient de faire coïncider les vérités de Moïse, d'Hermès, de Pythagore et de Platon. ${ }^{20}$ Il est évident que Le Caron voulait se présenter comme adepte de philosophie platonicienne et néo-platonicienne, et qu'il partageait avec ses contemporains humanistes leur syncrétisme chrétien. Dans cette perspective le vrai but de l'existence humaine, comme Le Caron l'a indiqué dans son dialogue sur la tranquillité d'esprit, s'identifie avec l'expérience de la contemplation intellectuelle. Dans sa Philosophie de 1555 Le Caron définit la philosophie comme "l'amour de sagesse, laquelle retirée des choses corporelles, contemple les divines, et recherche la verité". ${ }^{21}$ Pour lui la pensée de Platon a été "abastardie" par "Aristote, Xenocrate, Theophraste, Zenon, Epicure, et les autres Peripateticiens, Academiciens, Stoiciens, Epicuriens"'.22 Les titres des dialogues projetés du tiers livre des Dialogues (où figurent les noms d'Héraclite, de Parménide et de Pythagore) et plusieurs références dans sa Philosophie indiquent une familiarité avec la philosophie hermétique. Mais en bon syncrétiste chrétien, Le Caron suit l'opinion de Justin Martyr d'après qui la doctrine platonicienne remonterait à Moïse plutôt qu'aux "colonnes de Mercure".23 Son utilisation des sources, et surtout des dialogues de Platon, dans le Ronsard renforcera également l'idée qu'on pourrait se faire de l'orthodoxie philosophique de ce chrétien platonisant qui débute dans les cercles humanistes.

Regardons de plus près les arguments des quatre interlocuteurs et leur rapport avec cette vision essentiellement harmonieuse d'un univers plein d'analogies que Dieu a voilées au vulgaire. C'est à Ronsard qu'incombe l'honneur du premier et du plus long discours. Après avoir établi la primauté temporelle de la poésie relativement à la philosophie, il discutera du concept de l'imitation. Dès la première phrase il suscite le critère de "l'utilité publique" et justifie la poésie comme véhicule de sagesse et de vertu. La poésie ainsi que la philosophie se propose donc une fonction à la fois épistémologique et morale:

... de touts les secrets des choses plus cachées en nature, qui sont par-
venus aux hommes du moien eage et de cétui-ci, il n'en faut donner la
gloire à autre, qu'à la poësie. ... [Les poëtes] excitoient châcun à faire
les choses honnorables à l'immortalité de leurs noms. Ainsi leurs chants
estoient une vraie doctrine de bien-vivre, ou plustost une sainte fureur
qui inspiroit les hommes à congnoistre le lieu de leur celeste origine,
et dresser à ce but toutes leurs pensées. (129-129v)

Avant de procéder aux deux points principaux de son apologie, Ronsard allègue comme preuves de l'excellence de la poésie trois lieux communs en ce qui concerne son pouvoir affectif. La douceur de la lyre orphique a civilisé l'homme sauvage; la poésie console l'homme vertueux 
mais mortel en lui conférant l'immortalité; et, comme la peinture, elle plaît et enseigne par l'efficacité de son imitation de la nature. Cette comparaison traditionnelle depuis Horace entre la poésie et la peinture souligne la nécessité et de l'art et d'une "plus haulte science". Imitation et révélation vont de pair dans la poésie: "car outre le sujet qu'elle se propose à imiter, elle doit comprendre tout ce qui est d'exquis, de rare et de perfait es sciences non indignes de sa gravité" (130v). On voit déjà avec ces trois topoi que, dans un sens, l'utilité de la poésie vient de sa douceur. C'est à cause de son pouvoir affectif qu'elle apporte aux hommes les avantages de la civilisation, le renom éternel et une voie d'accès aux vérités cachées.

Pour faire preuve de la primauté de la poésie, Ronsard cite un certain nombre de passages tirés de Pindare ${ }^{24}$ qu'il compare à la pensée de Platon. Ce que ce philosophe a dit sur l'origine divine de la sagesse et de la vertu, sur l'omnipotence de Dieu, sur l'incertitude de la vie et la certitude de la mort, sur la faillibilité du vulgaire, Pindare l'avait exprimé avant lui. "Mais non seulement de cétui-ci, ains aussi de Theognide, Homere et infinis autres divins poëtes Platon a retiré le meilleur de sa philosophie" (132). Ce qui est antérieur n'est pas nécessairement supérieur. Mais Ronsard veut minimiser la contribution du philosophe pour agrandir celle du poète.

La doctrine de l'imitation ${ }^{25}$ qui attire l'attention de Ronsard dans le reste de son discours avait déjà été abordée dans la comparaison entre la poésie et la peinture. Et cette doctrine aussi, comme l'argument de la primauté temporelle de la poésie, est particulièrement bien accordée avec le rôle exalté que Ronsard réclame pour le poète, avec la fonction médiate qu'il attribue à la poésie et avec son concept élitiste du public:

\begin{abstract}
...comme toute les choses desirent de retenir leur perfection, et s'avilissent si quelquefois est diminué de leur excellence: ainsi les poëtes (lesquelz le peuple estimoit prophetes des Dieux) pensants estre indigne de prostituer leurs sacrées inventions au prophane vulgaire, les ont voulu couvrir de fables: afin qu'elles ne feussent entendues, que des plus sages et doctes. (132v)
\end{abstract}

Les fables jouent donc un rôle ambivalent dans la communication de la vérité à l'homme bien né. En sachant les utiliser, les poètes ont bien compris "la nature de l'esprit humain" qui "ravi et abstrait des pensements terrestres... cherche et recherche franchement, invente, conçoit, entend, traitte et dessigne infinis discours, pour trouver la verité, de laquelle la subtile fable lui donne quelque amorce" (133). Par l'intermédiaire de la fable poétique les "plus sages et doctes" éprouvent la "volupté" philosophique de la contemplation intellectuelle. Mais l'orne- 


\section{2 / Renaissance and Reformation}

ment de la fable ne plaît pas seulement à une élite:

J'admire non sans cause les poëtes, lesquelz se proposoient de contenter et les plus excellents et le commun. Car si les secrets cachez et enveloppez de leurs rares inventions ne pouvoient estre de châcun entendus: au moins le plaisir du discours chatouilloit et les uns et les autres, se rendant digne d'estre embrassé de touts. (133)

Le public du poète inclura donc "le commun"; et la disjonction de l'utile et de l'agréable est ressoudée comme chez Horace. Déguisée en "douce liqueur" la vérité passe, pourvu que les fables qui la représentent soient bien choisies. Les seules fables qui plaisent à Ronsard sont celles qui offrent "une exquise imitation de la nature des choses, et avec la delectation l'utilité conjointe" (133v).

Dans la dernière partie du discours Ronsard précise en quoi consisterait cette imitation. Ce faisant, il suscite les critères de la vraisemblance et de la bienséance:

Le poëte ... doit ... comme rapportant ses conceptions à l'université des choses discourir ce qui a peu estre fait, ou a esté vraisemblable, ou grandement necessaire, et le descrire de telle perfection, que rien ne soit en lui qui n'ait sa bienseance tant admirable, que la verité semble plustost l'avoüer sien que le fait mesme. (134)

A propos de cette phrase Grahame Castor remarque, à juste titre, qu'elle exprime la doctrine aristotélicienne de la mimésis quelques années avant que ce concept ne soit répandu en France, et qu'elle l'exprime de façon plus exacte que les écrits théoriques de Ronsard lui-même. ${ }^{26}$ Le concept de la vraisemblance est inséparable de celui de la bienséance. La grandeur du poète se révèle dans sa capacité de choisir parmi tous les sujets qui se présentent "un qui mieux convienne et semble estre né avec la mesme chose" (134v). Ronsard croit avoir démontré donc que le poète a été le devancier du philosophe dans la transmission de la sagesse, et que la poésie est une voie d'accès à toutes les connaissances divines et humaines par le truchement de ses fictions à la fois utiles à ceux qui savent les interpréter et agréables à tout le monde.

A cette exposition fidèle de la doctrine de l'imitation proposée par la Pléiade, Jodelle va apporter une explication de la suprématie intellectuelle et morale de la poésie - le concept de la fureur poétique et la fable des Muses. Le discours poétique "surpasse les vulgaires conceptions" en raison de son caractère inspiré qui lui permet de communiquer "les secrets des choses épandues par nature" (135v):

Qu'est-ce autre chose céte fureur, si non l'inspiration des Muses, laquelle souffle en l'ame pure et non souillée des vaines afections, une sainte chaleur qui l'embraze toute de divinité. (136) 
Inspiré des Muses, le poète sera capable de saisir les principes immuables d'harmonie qui gouvernent l'univers:

Qu'est-ce qui pourroit apporter plus seure et ferme congnoissance des choses divines et humaines, que la recherche de l'harmonie, qui entretient le monde: et fait que rien ne soit fortuit au ciel, ne rien en la terre, ne rien aussi es autres elements, sans cause et sans quelque raison de nombre et mesure? (138)

La cosmographie néo-platonicienne fournit à Jodelle des arguments en ce qui concerne les liens naturels entre la philosophie et la musique. Citant l'autorité de Pythagore, de Timée Locrien et de Platon, il constate qu'ils sont tous de l'opinion "que la musique est le plus excellent exercice de l'esprit pour accorder, composer et moderer les moeurs de l'ame en une gracieuse convenance, à l'imitation de l'ame de l'Univers" (138v). La recherche philosophique de la sagesse et de la vertu aboutira à la compréhension de la théorie pythagoricienne des nombres et de l'harmonie céleste dont la musique tempère les passions de l'homme. Mais la première musique, comme la première sagesse, est celle de la poésie: "Si plu diligemment nous voulons regarder, la Musique est la vraie philosophie, et la philosophie la vraie Musique: mais l'une et l'autre ont leur commencement de Poësie, ou plustost sont la mesme Poësie" (138v-139). Le mythe des Muses fait coda à ce discours élevé sur l'inspiration poétique.

Le Caron indique en marge les sources bien connues de ces pages sur la fureur poétique - le Phèdre et l'Ion de Platon. On pourrait signaler également l'Ode à Michel de l'Hospital de Ronsard et le Solitaire premier de Pontus de Tyard, publiés tous deux en 1552. Le poème de Ronsard contient une des exploitations les mieux connues du passage de l'Ion où Socrate dit au rhapsode que la poésie ne doit rien à l'art, et lui explique, avec l'image de l'aimant, la théorie de la fureur poétique. ${ }^{27}$ Le dialogue de Tyard contient un grand nombre de détails sur les Muses qui se retrouvent dans le dialogue de Le Caron - leurs origines, leur nombre, l'étymologie de Platon du mot "Muse", leur association aux sphères et leur rapport avec la musique. ${ }^{28}$ En somme, il paraît indubitable que le discours de Jodelle et celui de Ronsard reflètent fidèlement la pensée théorique des membres de la Pléiade, du moins en 1556.

Sans ambages, les deux orateurs, Pasquier et Fauchet, abordent le sujet du dialogue sous l'angle moral, sous l'angle de "l'utilité publique" à laquelle Ronsard avait fait allusion au début de son discours. Les discours de Pasquier apporteront quelques réserves à la justification de Ronsard; ceux de Fauchet, une réfutation. Pasquier, après avoir remercié les deux poètes du plaisir et de l'utilité de leurs discours, propose de chercher la raison pour laquelle Platon a banni les poètes, et même Homère, de sa République. Toute tentative de concilier la poésie et la 
philosophie devait faire face à ce jugement sévère de Platon qui dans d'autres passages de ses dialogues semble louer les efforts du poète. Pasquier utilise trois arguments dans sa défense d'Homère. D'abord, Platon lui-même recommande la sagesse d'Homère. Puis en reprenant l'argument de Jodelle, Pasquier suggère que "l'harmonie des sons celestes accordez d'un perpetuel mouvement a esté mieux exprimée par Homere, que par nul autre" (141). Finalement, Platon dans son Cratyle cite l'autorité d'Homère dans le domaine de l'onomastique. C'est au deuxième argument que Pasquier accorde la plus grande attention. Dans cette section de sa défence du poète grec il est clair qu'il utilise les Allégories d'Homère d'Héraclite, d'où il a tiré presque mot à mot ses observations sur le contenu allégorique des vers d'Homère, y compris la description du bouclier d'Achille. Il est curieux que Le Caron, toujours soucieux d'indiquer ses sources, n'ait pas cette fois reconnu sa dette. ${ }^{29}$

Après avoir conclu que chez Homère plus que chez nul autre "apparoissent les traces de toutes les sciences et les exemples des vertus pour bien et heureusement vivre en politique compagnie" (142v), Pasquier pense trouver l'explication du bannissement en comparant "le dessin de l'un avec l'intention de l'autre". Platon, en dressant le plan de sa République parfaite, a voulu procéder non pas de la réalité mais d'une conception intellectuelle, de "l'Idée de la Justice divine" (143). Les poètes, par contre, prennent la réalité comme point de départ:

[Les poëtes] imitent les moeurs des hommes, qui ont esté ou vertueux ou vicieux, et plus souvent se bigarrent selon les diverses opinions du vulgaire. Daventage Platon... s'est tousjours proposé de suivre une vraie et certaine raison, reputant indigne de chatouiller ses citoiens de je nesçai quelles blandices et amorces des fables, desquelles la jeunesse ne peut rapporter aucune utilité. (143v)

Pasquier hésite. Il ne peut pas blâmer Platon; il ne veut pas rejeter la Muse d'Homère "digne d'memoire immortelle, mais non à touts et n'en touts lieux convenable" (143v-144).

Fauchet, pourtant, est plus catégorique, et formule des objections à l'imitation poétique sur le plan moral comme sur le plan épistémologique. Les fables qui racontent la corruption morale n'ont aucune utilité dans une république où la formation de la jeunesse est d'une importance capitale. Il faut façonner l'enfant à une rigoureuse vertu et non au plaisir que pourraient lui apporter les fables. En plus, les allégories obscures gardent leur mystère pour l'enfant qui ne sait pas les interpréter. C'est le pouvoir affectif dont se vante la poésie qui est condamné par Fauchet, car "plustost elle donne place aux passions desordonnées, qu'elle les donte et reprime" (145v). En disciple rigide de Platon Fauchet attaque l'imitation comme une représentation qui est doublement éloignée de la vérité, et qui finit par l'obscurcir. Il termine sa diatribe en mettant en 
cause la liberté que réclament les poètes qui "ne trouvent rien, qui ne soit convenable à leur imitation: et ne donnent moins aux choses vituperables leur grace et bienseance, qu'aux louables et vertueuses" (146).

La brève réponse de Jodelle reprend le leitmotif patriotique déjà présent au début du dialogue, et dans le discours de Ronsard qui voulait éviter de faire tort à ceux de son âge "par trop grande admiration des anciens" (132v). Jodelle semble avoir accepté comme critère la bienséance lorsqu'il concède à Fauchet qu'on peut observer chez les anciens "quelques fautes", des fables "mal inventées, mal disposées et mal accommodées à l'euvre entrepris" (146v). Mais il offre l'espoir que les Français feront mieux. Quoique cet espoir réponde assez mal aux objections de Fauchet, l'argument patriotique contre les anciens fait partie intégrante de la doctrine de la Pléiade et trouvera son écho chez les "modernes" dans la fameuse querelle littéraire du XVIIe siècle.

Lorsqu'il reprend la parole, Pasquier donne raison dans une certaine mesure à Fauchet, mais il limite les critiques morales de celui-ci à ces fables anciennes qui racontent des absurdités inutiles au sujet des.Dieux, et qui ne conviennent pas aux hommes "congnoissants un seul et toutpuissant Dieu" (146v). Mais il refuse de réprouver le pouvoir affectif de l'imitation poétique et la liberté du poète en ce qui concerne le choix des sujets à imiter. "L'image et figure des choses . . . naïvement depeintes et representées de leurs vraies et non feintes couleurs" nous procurent un grand plaisir et nous "enseignent ... de leurs formes" (146v-147). L'utile et l'agréable sont de nouveau conjoints, et à ce double but de la poésie s'ajoute le critère de la bienséance, déjà invoqué par Ronsard, et par Le Caron lui-même dans sa présentation du dialogue:

Quand donc les poëtes descrivent quelques choses horribles, quelques faits tristes et miserables, quelques moeurs et afections vehementes, il ne faut tant regarder au sujet, qu'à la bienseance de l'art qui l'a diligemment exprimé.... On doit considerer en la Poësie ou les excellentes et admirables sentences desquelles elle est toute pleine, ou l'agensement et convenance des personnes et des choses accommodées à l'argument proposé. (147)

Pasquier répond à Fauchet que la poésie, en dépeignant le vice, peut nous encourager à le fuir aussi bien qu'elle peut nous faire aimer la vertu. Elle est donc "utile et à la temperance et à la haultesse d'esprit" (148$148 v$ ). Sans perdre de vue le critère de l'utilité morale de la poésie, Pasquier suggère qu'on la juge selon des critères internes esthétiques.

Fauchet tient ferme en face des justifications de Pasquier, et réitère les arguments en faveur de la censure. Dans l'intention avouée de plaire à ses lecteurs, le poète cherche nécessairement à exciter leurs passions. Il n'arrive pas à dépasser la nature qu'il imite. "Car quelz sont les dessins et estudes des hommes, telz aussi faut estre leurs esprits" (149). Fauchet 
rejette toute justification de l'imitation sur le plan moral et toute théorie transcendante de la poésie.

Brian Barron, qui base ses conclusions avant tout sur les discours de Pasquier et de Fauchet, soutient que le dialogue de Le Caron vise à remplacer la justification utilitariste de la poésie et la théorie de la fureur poétique par une justification esthétique: "Ultimately then, the dialogue must be seen as an appeal for honesty in literary criticism, for a justification of poetry which is based on the reality of the reader's response rather than on the supposed "divinity" of the art." 30 Il n'en est pas moins vrai que Le Caron a pris soin d'accorder au poète le privilège du dernier mot. Ronsard met fin à la discussion en revenant à la doctine de l'inspiration divine: "Céte poësie donc laquelle est inspirée d'une sainte fureur, ne peut rien imiter qui ne soit tout celeste, illustre et accompli" (149v). Il n'y a donc pas de synthèse finale. Ronsard ne répond ni aux réserves de Pasquier ni à l'intransigeance morale de Fauchet. Il ne fait que réaffirmer la haute valeur de la poésie qui est d'inspiration divine, avec la restriction que "touts ne sont également favorisez de Dieu" (149).

En conclusion, si on considère le dialogue dans son ensemble, on peut dire que Le Caron essaie avec les discours de Ronsard et de Jodelle de refléter assez impartialement les préoccupations théoriques des poètes de la Pléiade à l'époque de leur plus grand essor. A leurs justifications néo-platoniciennes de la supériorité de la poésie, il juxtapose les arguments d'ordre moral et esthétique que pourraient formuler deux orateurs qui connaissent bien leur Platon (et peut-être leur Aristote), mais qui ne partagent pas les mêmes vues sur la valeur de la poésie. La voix de l'Autre est assez bien installée dans le Ronsard. Ce dialogue semble incarner par sa forme et par les hésitations à l'intérieur de cette forme un moment historique plutôt ouvert, pendant lequel les humanistes cherchaient à incorporer de nouvelles idées à une vision toujours unifiée. Laissant au lecteur le droit de choisir son point de vue, Le Caron se contente de cerner les problèmes philosophiques que doit de tout temps affronter le poète sur la nature de son art.

McMaster University et London, England

Notes

1 Pour les détails biographiques sur Le Caron on consultera Lucien Pinvert, "Louis le Caron, dit Charondas," Revue de la Renaissance, 2 (1902), 1-9, 69-76, 181-188; et la thèse de F. Gohin, De Lud. Charondae (1534-1613): vita et versibus (Paris: Leroux, 1902). Les études de Pinvert et de Gohin proposent une date de naissance différente. Pinvert suggère 1536, citant les liminaires du Commentaire de la Coustume de Paris (1582), ouvrage juridique de Le Caron. La Monnoye, dans les notes qui suivent la notice sur Le Caron chez La Croix du Maine, 
Bibliothèque Françoise, nouv. éd. (Paris: Saillant et Nyon, 1772), semble donner raison au choix de cette date: “...par la légende qui est autour de son portrait, on voit qu'en 1579 il avait quarante-trois ans" (p. 47). La thèse de Gohin propose 1534. La validité de cette date se trouve appuyée même chez Pinvert qui parle d'une "inscription que les descendants ont fait placer, en 1854, dans l'église de Saint-Samson, à Clermont," à l'occasion de la mort de Le Caron, "le 18 septembre 1613, à l'âge de 79 ans" (Pinvert, p. 186). Pinvert trouve cette date contradictoire parce qu'elle n'est pas conforme à la date de naissance de 1536 , mais on voit qu'elle renforce la date proposée par Gohin. La date de 1534, établie par Gohin, est généralement acceptée.

Le Caron s'est donné une généologie ingénieuse. "Je vous prie . . . avoir tousiours souvenance de vostre Charondas, je dis Charondas, qui est mon nom propre et originaire, non imaginairement emprunté, comme aucuns l'ont voulu calomnier. Car ainsi qu'autresfois je vous ai monstré par escript, au voyage que fit Jean Paleologue Empereur de Constantinople, avec le Patriarch, vers le pape Eugene IIII, estoient plusieurs Grecs à sa suitte, et entre autres ce grand Bessario en la compagnie duquel estoit Charondas Autheur de nostre race ... : le nom de Charondas ayant esté du commencement changé en Charond, depuis en le Caron, pour la commodité de la prononciation." Voir Responses du droict françois, L. ix, resp. lxxvi, citée dans la thèse de Gohin, p. 2.

2 Catalogus legum antiquarum, una cum adjuncta summaria interpretatione, per J. Ulricum Zasium Brigantinum diligenter collectus, cum annotationibus Ludovici Charondae (Paris: G. Cavellat, 1554).

3 La Poësie de Loys le Caron Parisien (Paris: V. Sertenas, 1554; Paris: G. Cavellat, 1554; Paris, G. Robinot, 1554).

4 La Claire ou de la Prudence de droit, dialogue premier, plus la Clarté amoureuse (Paris: G. Cavellat, 1554; Paris: G. Corrozet, 1554).

5 La Philosophie de Loys le Caron (Paris: J. Longis, 1555; Paris: G. LeNoir, 1555; Paris: V. Sertenas, 1555).

6 "Parce que la grandeur des dialogues ensuivants de la beauté, et des autres livres merite plus ample volume, on a differé de les imprimer jusques à ce que l'auteur aiant plus grande commodité mette touts ses euvres en lumiere" (Dialogues, p. 177).

7 Brian Barron, "Poet and Orator in Louis le Caron's 'Dialogue de la poësie (1556)," dans French Renaissance Studies, 1540-70: Humanism and the Encyclopedia, ed. Peter Sharratt (Edinburgh: Edinburgh Univ. Press, 1976), pp. 21-35.

8 Voir surtout Mustapha Kemal Bénousi, Le Dialogue philosophique dans la littérature française du seizième siècle (The Hague/Paris: Mouton, 1976) et Eva Kushner, "Le Dialogue en France au XVIe siècle: quelques critères génologiques," Canadian Review of Comparative Literature/ Revue Canadienne de Littérature Comparée, 5 (1978), 141-153.

9 Bénouis, pp. 141-143.

10 Kushner, p. 141.

11 Ibid., pp. 145-146.

12 Ibid., pp. 152-153.

13 Sur les rapports entre Fauchet et Pasquier voir J.G. Espiner-Scott, "Claude Fauchet et Estienne Pasquier," Humanisme et Renaissance, 6 (1939), 352-360.

14 Estienne Pasquier, Choix de lettres sur la littérature, la langue et la traduction, éd. D. Thickett (Genève: Droz, 1956), pp. 5-6.

15 Pasquier, Les Recherches de la France. Reveuës et augmentées d'un Livre, et de plusieurs chapitres par le mesme Autheur (Paris: Laurent Sonnius, 1607), p. 868. Un autre chapitre du même livre, où Pasquier commente certains néologismes de son époque, contient une allusion assez curieuse: "De mon temps, i'ay veu plusieurs mots mis en usage, qui n'estoient recogneus par nos devanciers. . . .Le premier qui mit en oeuvre Avant-propos, pour Prologue, fut Louys le Charond en ses Dialogues, dont on se mocquoit du commencement: Et depuis ie voy ceste parole receuë sans en douter: Non sans cause" (p. 955).

16 Kushner, p. 148.

17 Du Bellay, La Deffence et illustration de la langue francoyse, éd. H. Chamard (Paris: Didier, 1961). Du Bellay dit que "Le poëte et l'orateur sont comme les deux piliers qui soutiennent 


\section{8 / Renaissance and Reformation}

l'edifice de chacune Langue" (pp. 87-88). Vers la fin de ce manifeste de la Pléiade, paru en 1549 , il souligne surtout la valeur affective de la poésie: "celuy sera veritablement le poëte que je cherche en nostre Langue, qui me fera indigner, apayser, ejouyr, douloir, aymer, hayr, admirer, etonner, bref, qui tiendra la bride de mes affections, me tournant ça et la à son plaisir"' (p. 179).

Cependant, Ronsard fait bien la distinction entre l'orateur et celui qui s'inspire des Muses dans l'Ode à Michel de l'Hospital, où Jupiter déclare que l'orateur plaide par art, mais que les Muses n'en auront pas besoin (antistrophe et épode 12).

Sur les rapports entre la poésie et la rhétorique voir E.R. Curtius, European Literature and the Latin Middle Ages, tr. W.R. Trask (New York: Harper Torchbooks, 1963), pp. 145-166 et B. Munteano, Constantes dialectiques en littérature et en histoire (Paris: Didier, 1967), pp. 150 et seq.

18 J.G. Espiner-Scott, dans sa biographie de Fauchet, Claude Fauchet: sa vie et son oeuvre (Paris: Droz, 1938), remarque dans sa description du dialogue de Le Caron que ce bruit du palais de justice était notoire à l'époque.

19 Michel Foucault, Les Mots et les choses (Paris: Gallimard, 1966), pp. 32-59.

20 Voir, par exemple, les ouvrages de Curtius, de D.P. Walker, The Ancient Theology: Studies in Christian Platonism from the Fifteenth to the Eighteenth Century (Ithaca, N.Y.: Cornell Univ. Press, 1972), pp. 1-41, 63-131, et de Frances A. Yates, The French Academies of the Sixteenth Century (London: Warburg Institute, 1947). Frances Yates souligne bien l'importance de la pensée ficinienne en ce qui concerne les rapports entre la philosophie et la religion (pp. 2-6).

21 Le Caron, La Philosophie, p. 10v.

22 Ibid., pp. $17 \mathrm{v}-18$.

23 Ibid., pp. $22 \mathrm{v}$ et 53.

24 Ronsard, depuis la publication de ses Odes en 1550, passait pour le Pindare français. Cette série de citations, toujours identifiées dans les notes marginales de Le Caron, permet à Ronsard et à Le Caron d'étaler une connaissance précise de Pindare.

25 Sur les concepts de l'imitation voir Grahame Castor, Pléiade Poetics: A Study in SixteenthCentury Thought and Terminology (Cambridge: Cambridge Univ. Press, 1964) et Henri Weber, La Création poétique en France au XVIe siècle (Paris: Nizet, 1955), pp. 107-160.

26 Castor, pp. 58, 61-62. Henri Weber constate que: "La Poétique d'Aristote... ne commence à exercer une influence en France qu'à partir de 1561 par l'intermédiaire de la poétique latine de Jules Cesar Scaliger, qui en diffuse les principes" (p. 124).

27 Mais, comme le remarque Paul Laumonier dans son édition critique du poète, "Ronsard laisse de côté, consciemment ou non, l'intention ironique de Platon, qui semble avoir échappé à ses contemporains, comme au rhapsode lui-même." Ronsard, Oeuvres complètes (Paris: Hachette, 1921), III, p. 142, n. 1.

28 Pontus de Tyard, Solitaire premier, éd. S. Baridon (Genève: Droz, 1950), surtout pp. 28-30, 39-40. Frances Yates dans The French Academies commente les sources des idées de Tyard dans le Solitaire premier. Quant à la fureur poétique, Tyard suit de près les commentaires de Marsile Ficin sur Platon (p. 81). Les détails sur les Muses, leur nombre - trois à l'origine, puis neuf - et leur association à l'anima mundi et aux sphères sont empruntés à deux mythologues de la Renaissance: L.G. Giraldi, De deis gentium et Heinrich Loriti, dit Glareanus, Dodecachordon (pp. 83, n. 2 et 133). Sur l'importance de la musique des sphères comme topos de la poésie pendant la Renaissance, on consultera S.K. Heninger, Jr., Touches of Sweet Harmony: Pythagorean Cosmology and Renaissance Poetics (San Marino, Calif.: Huntington Library, 1974), pp. 179 seq.

29 L'édition aldine des Allégories d'Homère a été publiée en 1505; l'édition de Gesner, avec la première traduction en latin, a paru à Bâle en 1544. L'auteur mal connu de cet ouvrage si important pour l'exégèse homérique (il ne s'agit pas du philosophe Héraclite) a recours à l'invective plutôt qu'à la logique pour défendre Homère contre l'opprobre de Platon: "Nous avons le droit de conclure: récits d'Homère, biographies de héros; dialogues de Platon, amours de garçons." Héraclite, Allégories d'Homère, éd., et tr. par F. Buffière (Paris: Belles Lettres, 1962), 78, p. 85.

30 Barron, p. 34. 